\title{
New technique to drive the central frequency and to improve bandwidth of EBG structures
}

\author{
Mohammad El Ghabzouri, Abdenacer Es Salhi \\ LETAS Dept. of physics, Faculty of Sciences, Mohamed I \\ University \\ Oujda, Morocco \\ elghabzouri.mad@gmail.com
}

\author{
Paulo M. Mendes \\ Dept. of Industrial Electronics, University of Minho \\ Guimarães, Portugal
}

\begin{abstract}
In this paper, a parametric study was done to find out the superstrat influence on locating the central frequency of the band gap. The present work compares the standard mushroom like with the EBG structures which are located between the substrate and the superstrat. The main motivation is to present a theoretical contribution by comparing the equations describing the central frequency of the band gap. In that way, this work investigates a new proposed design to shift the central frequency of the forbidden band to a low frequency, by inserting meandered lines to connect each part of the unit cell, which give an added capacitance to the EBG structures, in order to reach a low profile and lightweight EBG structure. The band gap was recognized by computing the transmission coefficient S21 resorting to the suspended line method (SLM).
\end{abstract}

Keywords-Low profile EBG; Suspended line method; electromagnetic band gap with circular slot (EBG-CS).

\section{INTRODUCTION}

The high spectrum of applications for EBG structures, not only in the antenna domain but also in other fields, the importance of the EBG structures in wireless and antenna applications, and the great need of technological advancement, drives researchers to find innovative ideas to improve the behaviour and performances of these structures, and to develop a low profile EBG structure that may benefit several wireless devices, In fact, our previously fabricated EBG structure with circular slots EBG-CS, and also the fabricated printed dipole antenna [1], made us wonder about new techniques to control the capacitance, in order to shift the band gap wherever and however a given device requires. Some works introduce a kind of slots on mushroom-like EBG to reach dual band gap below $10 \mathrm{GHz}$ [2]. The main works studied that modify Sievenpiper structure, which is mushroom- like [3] are resumed in the following work, including some advances in technologies resorting to EBG structures [4]. A majority of those works attempts to propose modifications to this basic Sievenpiper structure, whether to achieve different antenna parameters enhancement or dual band and low profile EBG antenna [1]. Recently, a few works show how efficient it is to reduce the specific absorption rate (SAR) using these EBG structures [1][5].

\section{THEORITICAL CONTRIBUTION}

A. Some existing equations for analytic identification of EBG structures band gap

First, we are going to focus our study on some proposed equation existing in the literature, in order to investigate how accurate they are at identifying the central frequency of the standard EBG mushroom's forbidden gap, and then the proposed EBG-CS (circular slot) structure. The EBG square patches are similar to an LC circuit [6], as demonstrated in Fig.1, In [6], the authors presented the equations (1) and afterward, in [7-8], modified equations (2) and (3), respectively, were presented.

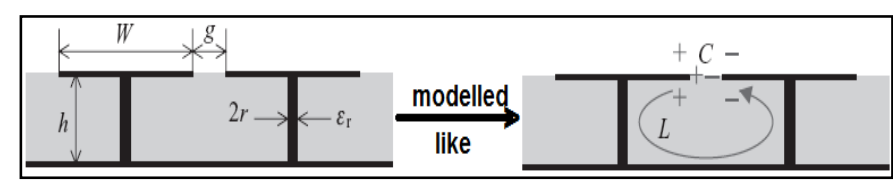

Fig. 1. The electrical equivalent circuit of EBG cells.

$$
\begin{gathered}
\text { (1) }\left\{\begin{array}{c}
L=\mu_{0} * h \\
C=\frac{W * \varepsilon_{0}\left(\varepsilon_{r}+1\right)}{\pi} \cosh ^{-1}\left(\frac{W+g}{g}\right)
\end{array}\right. \\
\text { (2) }\left\{\begin{array}{c}
L=\mu_{r} * \mu_{0} * h \\
C=\varepsilon_{0}\left(\varepsilon_{r}+1\right) \frac{(W+g)}{\pi} \ln \cos \left(\frac{\pi * g}{2 *(W+g)}\right) \\
L=\mu_{0} * h \\
C=C_{f}+C_{A}=\frac{W * \varepsilon_{0}\left(\varepsilon_{r}+1\right)}{\pi} \cosh ^{-1}\left(\frac{2 * W+g}{g}\right)+\frac{\varepsilon_{0} * \varepsilon_{r} * A}{h}
\end{array}\right.
\end{gathered}
$$


TABLE I. THEORITICAL COMPUTATION

\begin{tabular}{|l|c|c|c|}
\hline \multicolumn{1}{|c|}{ Parameters } & $\boldsymbol{L}(\boldsymbol{H})$ & $\boldsymbol{C}(\boldsymbol{F})$ & $\boldsymbol{F}(\boldsymbol{G H z})$ \\
\hline Equation (1) & $1.596 * 10^{-9}$ & $0.479 * 10^{-12}$ & 5.75 \\
\hline Equation (2) & $1.596 * 10^{-9}$ & $-1.43 .10^{-15}$ & 105 \\
\hline Equation (3) & $1.596 * 10^{-9}$ & $1.811 * 10^{-12}$ & 2.95 \\
\hline
\end{tabular}

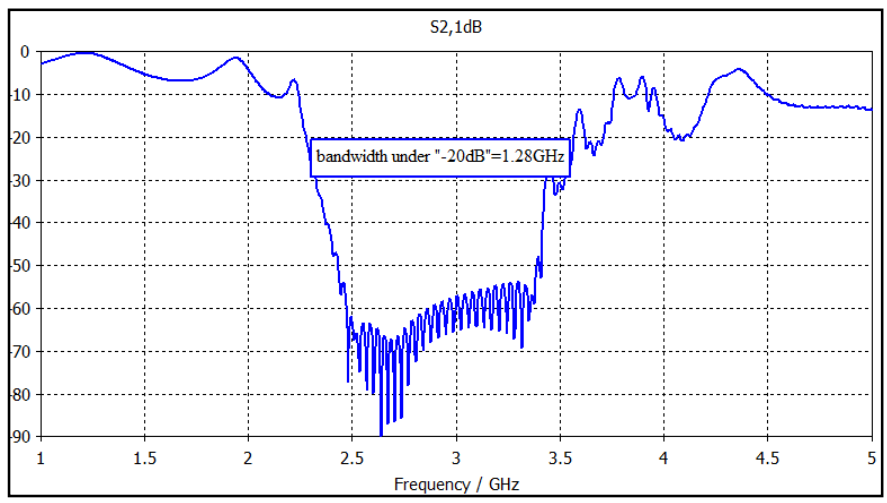

Fig. 2. The computed S21 results when using standard mushroom-like "without superstrat".

The simulated results presented in fig. 2 show a very good agreement between central frequency given by the third equation and the central frequency of the computed band gap, which is $2.9 \mathrm{GHz}$. Additionally the computed S21 shows remarkable improvement in the forbidden band bandwidth, as the bandwidth in [7] was $880 \mathrm{MHz}$, while the proposed structure presented $1.28 \mathrm{GHz}$, which translates into a bandwidth improvement of $45 \%$ comparatively to [7].

\section{B. Proposed equation for EBG and EBG-CS structures}

In this section, we would like to establish a new equation capable of exactly determining the desired band gap's resonance frequency, for EBGs mounted with both substrate and superstrat, as shown in Fig.3. For that goal, we present the equation for those kinds of EBG structures in (4).

(4) $\left\{\begin{array}{c}L=\mu_{0} *\left(h_{\text {sub }}+h_{\text {sup }}\right) \\ C=C_{f}+C_{A}=\frac{W * \varepsilon_{0}\left(\varepsilon_{r}+1\right)}{\pi} \cosh ^{-1}\left(\frac{2 * W+g}{g}\right)+\frac{\varepsilon_{0} * \varepsilon_{r} * A}{\left(h_{\text {sub }}+h_{\text {sup }}\right)}\end{array}\right.$

"A " represents the surface occupied by the via and circular slots when using our previously fabricated EBG-CS structure, $\mathrm{C}_{\mathrm{f}}$ is the capacitor between two adjacent patchs, and $\mathrm{C}_{\mathrm{A}}$ is the patch area capacitor.

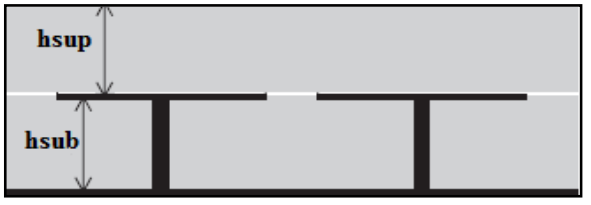

Fig. 3. The used concept of EBG and the proposed EBG-CS with superstrat

While $\mathrm{h}_{\text {sub }}$ and $\mathrm{h}_{\text {sup }}$ are the substrate and superstrat heights respectively, $\mu_{0}$ is the permeability in free space. As previously mentioned, we aim to study EBG and EBG-CS with meandered lines, that are mounted with and without superstrat, in order to allow a comparison of results.

The present chapter aims, first of all, to study the superstrat's influence on EBG behaviour, when used on top of the structure. Then we will propose a new technique to drive or control the capacitance modelled by the gap between cells, in order to enhance low profile EBG by driving the band gap to lower frequencies. This technique is based on uploading symmetric meandered lines on the EBG-CS cell, in order to obtain an added capacitance around the EBG-CS cells. We have already shown with this fabricated EBG-CS, the low profile and high efficiency of a proposed printed dipole antenna above this structure [1].

The unit cell band gap characterization was performed by computing the transmission coefficient S21 using the $50 \mathrm{ohm}$ suspended line method. Table 2 shows a comparison between the analytic central frequency of the band gap, obtained by using equation and the simulated central frequency. The results presented in Fig.4, show a very important behaviour, driving the band gap $0.5 \mathrm{GHz}$ lower (from $2.9 \mathrm{GHz}$ to 2.4 $\mathrm{GHz}$ ).

TABLE II. THEORITICAL COMPUTATION

\begin{tabular}{|l|c|c|c|}
\hline \multicolumn{1}{|c|}{ parameters } & $\boldsymbol{L}(\boldsymbol{H})$ & $\boldsymbol{C}(\boldsymbol{F})$ & $\boldsymbol{F}(\boldsymbol{G H z})$ \\
\hline Equation (4) & $3.129 * 10^{-9}$ & $1.386^{*} 10^{-12}$ & 2.41 \\
\hline Simulation & $* * * * * * *$ & $* * * * * * *$ & 2.40 \\
\hline
\end{tabular}

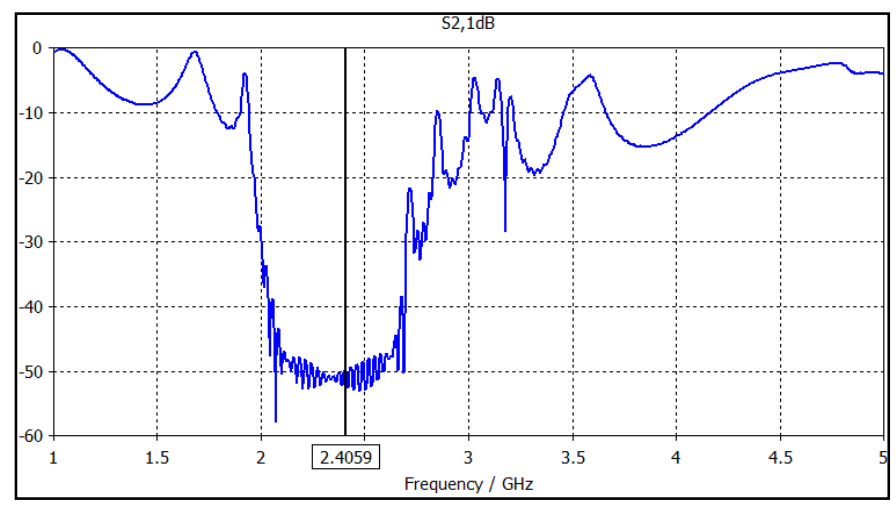

Fig. 4. S21 results when using superstrat. 


\section{SUPERSTRAT PERMITIVITY INFLUENCE ON BAND GAP}

Before we begin, we underline that there are many available methods to identify the band gap of EBG structures, whether numerical, analytic, simulation methods such as finite difference time domain FDTD. But in fact, the most practical and the fastest method in simulations is the $50 \mathrm{ohm}$ suspended line method. The criterion of this method is based on inserting a conducting line on top of the substrate, and then placing it on top of the modelled EBG structure. Finally, we feed both sides of the whole structure through a wave guide port, as demonstrated in the figure bellow.

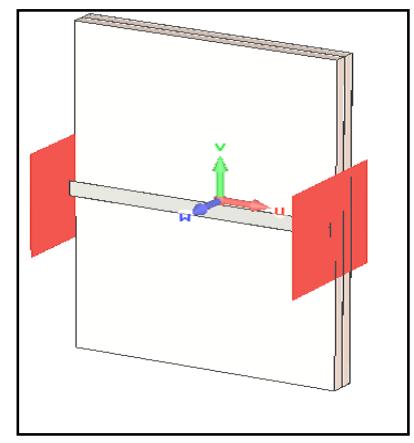

Fig. 5. Suspended line design with EBG structure in addition to two wave guid ports.

We clarify, among the chapters bellow, the influence of both the superstrat's permittivity, and its height on the transmission coefficient results.

\section{A. Superstrat permitivity}

The previous proposed EBG-CSs were fabricated using Rogers RT6010, with dielectric constant 10.2. The results are illustrated in the Fig.6, showing the effect of this parameter on the transmission coefficient S21. Both studied superstrat and substrate materials' thicknesses were fixed at $1.27 \mathrm{~mm}$.

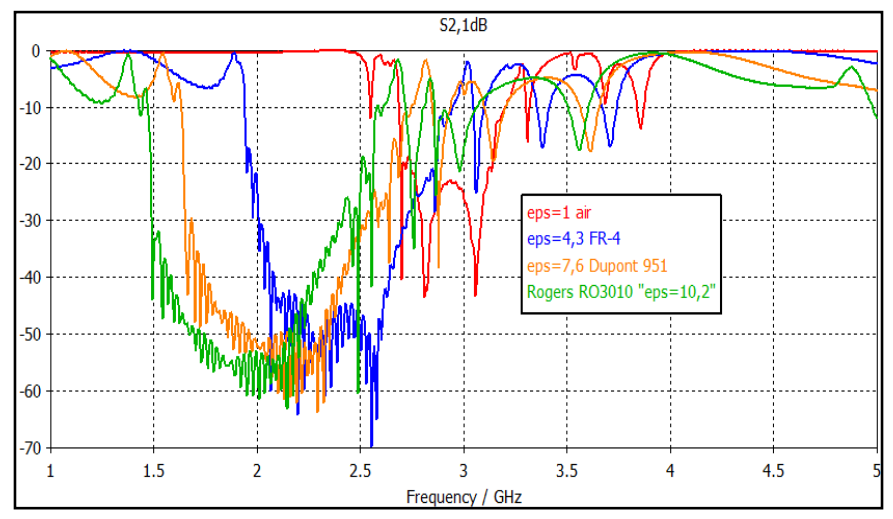

Fig. 6. Compared S21 results when varying the superstrat material

\section{B. Superstrate height}

In this following study we fix the superstrat as RO3010, and we will investigate the S21 by tuning the height of the superstrat, in order to identify the optimum result. Results are presented in Fig.7.

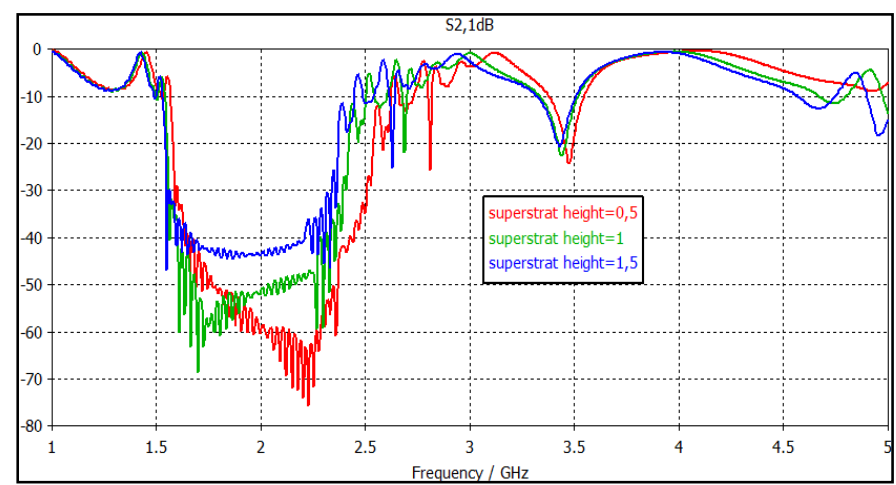

Fig. 7. The simulated results showing the height's superstrat influence on band gap.

\section{Results and discussion}

The obtained results show that the use of superstrat materials which have lower permittivity is translated into a shift of the band gap into higher frequencies and a narrowing of its bandwidth. Fig.7 shows that the optimum result is met when the height of superstrat and substrate are equal.

\section{CAPACITANCE DRIVING BY USING MEANDERED LINES}

In the following chapter, we present an innovative technique that proved its efficiency in our simulations. It consists in driving the band gap's central frequency to lower frequencies, in order to have low profile devices. The mushroom-like EBG standard square patches and their electric model LC circuit are a well known. The concept of this new proposed design aims to increase the distance of the four lines connecting each part of our EBG-CS cell, as shown in Fig.8. This technique showed a very important behaviour which manifests itself in an increase of the forbidden band's bandwidth, well as shifting the band gap to lower frequencies. Fig. 9 describes how we could drive the central frequency from $2.9 \mathrm{GHz}$ to $1.9 \mathrm{GHz}$ by increasing the lines' length.

Fig.8. shows the design of the EBG-CS with meandering lines, accompanied by its modified unit cell design with all sizes. Additionally, the computed results of the transmission coefficient S21 of the EBG-CS structure are illustrated in Fig.9. 

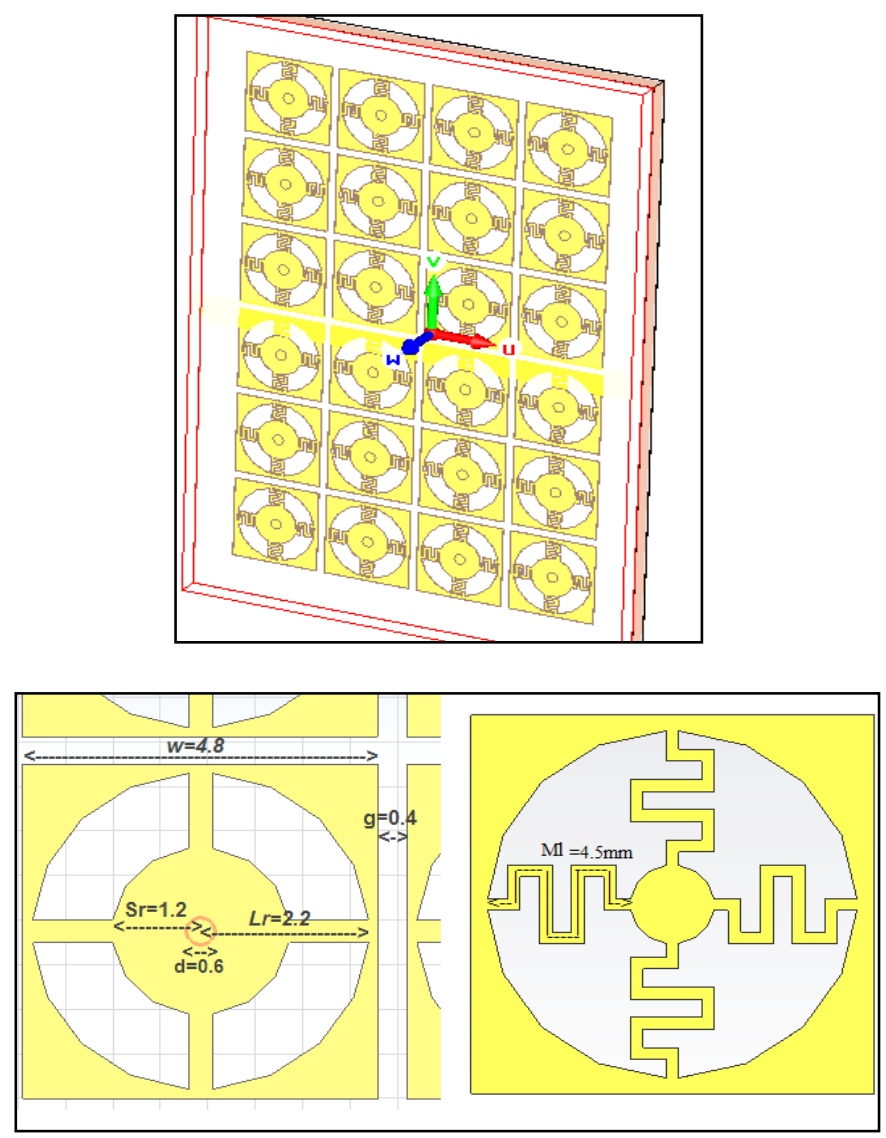

Fig. 8. Design of the whole EBG-CS structure with meandered line on the top, accompanied with modified unit cell details.

$\mathrm{W}=4.8 \mathrm{~mm}, \mathrm{~g}=0.5 \mathrm{~mm}$ (gap between cells), Small radius $\mathrm{Sr}=1.2 \mathrm{~mm}$, large radius $\mathrm{Lr}=3.2 \mathrm{~mm}$, via radius $\mathrm{Vr}=0.5 \mathrm{~mm}$, $\mathrm{h}=3 \mathrm{~mm}$.

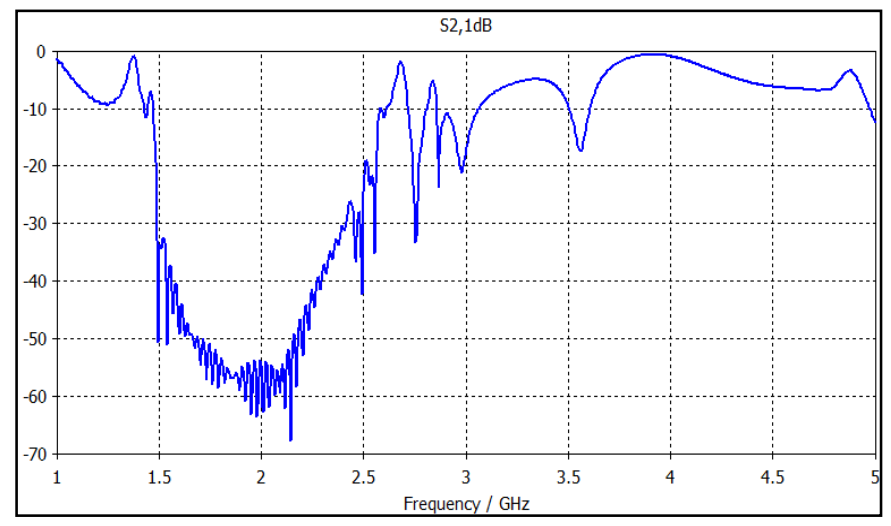

Fig. 9. The obtained S21 parametre of the antenna used with the proposed EBG-CS with meandered lines.
This new technique achieves our main goal, which is the improvement of the forbidden bands' bandwidth, and especially having low profile devices, by presenting a small EBG structure which could have a band gap on low frequency. The present structure could be highly recommended to several other fields in addition to the antenna community.

\section{CONCLUSION}

The main goal of this paper is to present a practical guide, in order to reach a perfect control of the forbidden band of EBG structures, together with improving its bandwidth. At the beginning we showed a $45 \%$ bandwidth improvement of the studied EBG comparatively to literature. Also, with this paper, we had a theoretical contribution by establishing an equation that identifies the forbidden band, favour to the EBG-CSs. The innovative idea of this work is about driving the band gap of EBG structures to lower frequencies by increasing the length of the lines connecting each part of the proposed unit cell. These meandered lines achieved remarkable results, having driven the forbidden band $1 \mathrm{GHz}$ lower, which is significant result to have low profile EBG structures.

\section{References}

[1] Mohammad El Ghabzouri, Abdenacer Es Salhi, Paulo M. Mendes, "Dual band antenna size reduction using EBG structure,"medICT conferences 2015 Saidia, "unpublished".

[2] Osman Ayop, Mohamad Kamal A. Rahim and Thelaha Masri "A Dual Band Gap Slotted Patch Electromagnetic Band Gap for Dual Band Microstrip Antenna," 2008 ieee international RF and microwave conference proceedings.

[3] D. Sievenpiper, L. Zhang, R. F. J. Broas, N. G. Alexópolous, and E. Yablanovitch, "High-Impedance Electromagnetic Surfaces with a Forbidden Frequency Band," IEEE Transactions on Microwave Theory and Techniques, Vol.47, No.11, November 1999, pp. 2059-2074.

[4] Alka Verma, "ebg structures and its recent advances in microwave antenna," International Journal of Scientific Research Engineering \& Technology (IJSRET) Volume 1 Issue 5 pp 084-090 August 2012.

[5] Kwok-Hung Chan, Member, IEEE, Ryo Ikeuchi, and Akimasa Hirata, Senior Member, IEEE "Effects of Phase Difference in Dipole PhasedArray Antenna Above EBG Substrates on SAR" IEEE Antennas Wireless Propag. Lett., vol. 12, 2013.

[6] F. Yang, Y. Rahmat-Samii, "Electromagnetic band gap structure in antenna engineering" Cambrige university press, 2008.

[7] M. S. Alam1, M. T. Islam, and N. Misran, "a novel compact splitring slotted electromagnetic band gap structure for microstrip patch antenna performance enhancement" Progress In Electromagnetic Research, vol. 130, 2012.

[8] Osman Ayop, Mohamad kamal A rahim, “Analysis of mushroom-like electromagnetic band gap structure using suspended transmission line technique" 2011 ieee international RF and microwave conference proceedings RFM2011, $12^{\text {th }}-14^{\text {th }}$ december 2011 , Seremben, Malaysia 